\title{
Alpha-particle physics in the tokamak fusion test reactor DT experiment
}

\author{
S J Zweben ${ }^{a}$, V Arunasalam ${ }^{a}$, S H Batha ${ }^{b}$, R V Budny ${ }^{a}$, C E Bush ${ }^{c}$, \\ S Cauffman ${ }^{a}$, C S Chang ${ }^{d}, \mathrm{Z} \mathrm{Chang}^{a}$, C Z Cheng ${ }^{a}$, D S Darrow ${ }^{a}$,

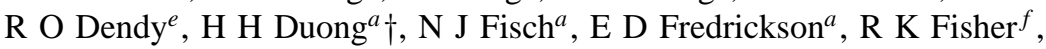 \\ R J Fonck ${ }^{g}, \mathrm{G} \mathrm{Y} \mathrm{Fu}{ }^{a}$, V Goloborod'ko ${ }^{h}$, N Gorelenkov ${ }^{i}, \mathrm{R} \mathrm{J}$ Hawryluk $^{a}$,

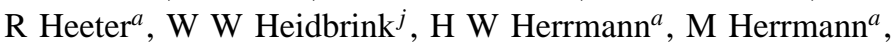 \\ D W Johnson ${ }^{a}$, J Machuzak ${ }^{k}$, R Majeski ${ }^{a}, \mathrm{~K}$ M McGuire ${ }^{a}, \mathrm{G} \mathrm{McKee}^{g}$,

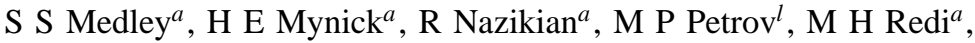 \\ S Reznik ${ }^{h}$, J Rogers ${ }^{a}$, G Schilling ${ }^{a}$, D A Spong ${ }^{c}$, J D Strachan ${ }^{a}$,

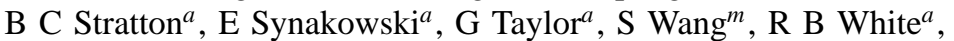 \\ $\mathrm{K} \mathrm{L} \mathrm{Wong}^{a}$, V Yavorski ${ }^{h}$ and the TFTR Group \\ ${ }^{a}$ Princeton Plasma Physics Laboratory, P O Box 451, Princeton, NJ 08543, USA \\ ${ }^{b}$ Fusion Physics and Technology, Torrance, CA 90503, USA \\ ${ }^{c}$ ORNL, Oak Ridge, TN 37831, USA \\ ${ }^{d}$ Courant Institute, New York University, New York, NY 10012, USA \\ ${ }^{e}$ UKAEA/Euratom, Culham, Abingdon, UK \\ ${ }^{f}$ General Atomics, San Diego, CA 92186, USA \\ ${ }^{g}$ University of Wisconsin, Madison, WI 53706, USA \\ ${ }^{h}$ Institute for Nuclear Research, Kiev, Ukraine \\ ${ }^{i}$ TRINITI, Troisk, Moscow, Russia \\ ${ }^{j}$ University of California, Irvine, CA 92717, USA \\ ${ }^{k}$ MIT, Cambridge, MA 02139, USA \\ ${ }^{l}$ Ioffe Institute, St Petersburg, Russia \\ ${ }^{m}$ Institute of Plasma Physics, Academia Sinica, Hefei, China
}

\begin{abstract}
A summary is presented of recent alpha-particle experiments on the tokamak fusion test reactor. Alpha particles are generally well confined in MHD-quiescent discharges, and alpha heating of electrons has been observed. The theoretically predicted toroidicity-induced Alfvén eigenmode has been seen in discharges of $\leqslant 1 \mathrm{MW}$ of alpha power, but only in plasmas with weak magnetic shear.
\end{abstract}

\section{Classical alpha confinement and thermalization}

Alpha-particle heating is the basis for ignition in DT tokamaks, so it is important to understand the confinement and stability of energetic alphas before building a power plant based on this reaction. Efficient alpha-particle heating requires that almost all the $3.5 \mathrm{MeV}$ alpha-particle power (i.e. $20 \%$ of the total fusion power) should be transferred to the thermal plasma before it is lost to the vessel wall. Therefore, the alpha-particle confinement time needs to be longer than the collisional alpha thermalization time, which itself is very much longer than the alpha transit time. For example, the alpha transit time in the tokamak fusion test reactor (TFTR) is $\tau_{\text {trans }}=2 \pi R / v_{\alpha} \approx 10^{-6} \mathrm{~s}$, while the alpha thermalization time is $\tau_{\text {therm }} \approx 0.3 \mathrm{~s}$, with $R=2.5 \mathrm{~m}, I_{\mathrm{p}}=2.5 \mathrm{MA}, T_{\mathrm{e}}(0)=10 \mathrm{keV}$ and $n_{\mathrm{e}}(0)=10^{14} \mathrm{~cm}^{-3}$.

$\dagger$ General Atomics ORAU fellow at PPPL. 
Since the canonical angular momentum of a collisionless alpha particle is an adiabatic invariant in a toroidally symmetric tokamak, alphas which are confined on their first orbit should remain well confined, at least in the absence of non-axisymmetries. The fraction of alphas lost to the wall on their first orbit depends mainly upon the plasma current, which determines the confining poloidal magnetic flux and the size of the trapped 'banana' alpha orbits. Only $\leqslant 5 \%$ of alphas are lost on their first orbit for plasma currents above $I_{\mathrm{p}} \geqslant 2.5 \mathrm{MA}$ (i.e. at TFTR current).

In the presence of Coulomb collisions, a constant alpha source relaxes to a steady-state 'slowing-down' distribution, $f_{\alpha} \propto 1 / v_{\alpha}^{3}$ between the birth energy of $3.5 \mathrm{MeV}$ and the critical energy of approximately $0.3 \mathrm{MeV}$, below which ion drag dominates electron drag. However, these collisions cause little high-energy alpha transport, since the alpha thermalization time is long and the rate of pitch-angle scattering into different types of orbits is small compared with the thermalization rate. For example, on TFTR, the alpha pitch-angle scattering time $\tau_{\text {scatt }} \approx 5-10 \mathrm{~s} \gg \tau_{\text {therm }}$.

The experiments on alpha confinement in TFTR have tested this classical collisional model and are beginning to identify some non-axisymmetries responsible for deviations from this classical behaviour. Alpha-particle confinement TFTR has been measured using several unique alpha-particle diagnostics developed at PPPL over the past 10 years.

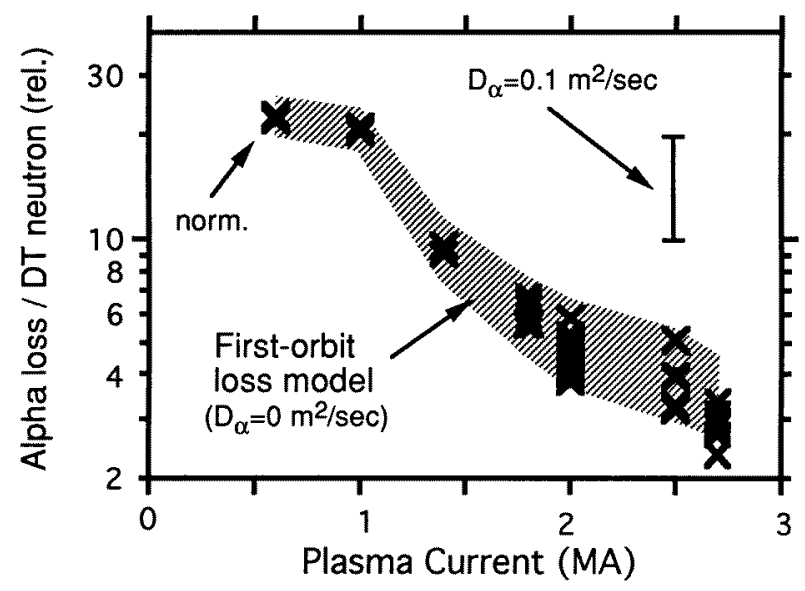

Figure 1. Alpha-particle loss from TFTR as measured by a scintillation detector located at the vessel bottom in the ion grad- $B$ drift direction. The alpha loss is normalized by the global DT neutron emission and decreases with increasing plasma current, as expected from the classical first-orbit loss model. The bar at $I=2.5 \mathrm{MA}$ represents a calculation showing the expected signal level, assuming a diffusion rate of counter-passing alphas of $\mathrm{D}_{\alpha} \approx 0.1 \mathrm{~m}^{2} \mathrm{~s}^{-1}$, which implies that the alpha diffusion rate is very low.

Alpha-particle loss to the vessel wall has been measured with a set of imaging scintillator detectors similar to pinhole cameras. The behaviour of alpha loss in TFTR as a function of plasma current is shown in figure 1 for the detector at the vessel bottom. The alpha loss rate (normalized by the neutron source rate) decreases with increasing plasma current, as expected from the classical first-orbit loss model, and the lost alpha pitch angle, energy, and time dependences are also consistent with this model [1]. This result sets a very low upper limit of $D \leqslant 0.1 \mathrm{~m}^{2} \mathrm{~s}^{-1}$ for the radial diffusion rate of confined counter-passing alphas near the plasma centre, which is similar to diffusion coefficients measured for other fast ions in MHD-quiescent tokamaks [2]. 
The confined alphas inside the TFTR plasma have been directly measured using two different charge-exchange techniques. The first, pellet charge exchange (PCX), uses the ablation cloud of an injected lithium or boron pellet to doubly charge exchange the alphas, which are then detected outside the vessel with an energy analyser. The second, AlphaCHERS, uses the neutral beam atoms to singly charge exchange the alphas, which are then detected as $\mathrm{He}^{+}$ions using optical emission spectroscopy. Owing to geometrical diagnostic constraints, PCX measures only trapped alphas with pitch angles nearly perpendicular to the toroidal field, while Alpha-CHERS measures both passing and trapped alphas.

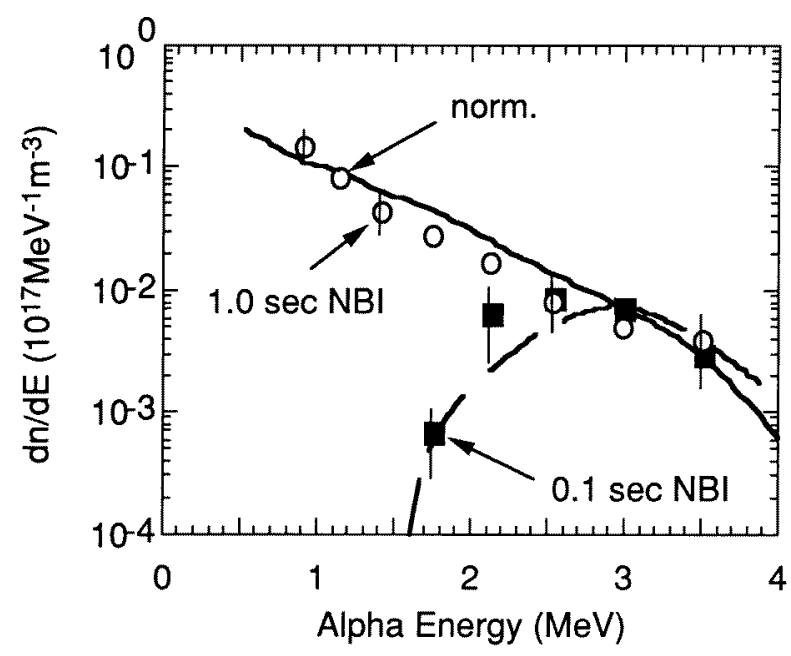

Figure 2. Energy spectra of confined alphas near the centre of TFTR as measured by the PCX diagnostic just after two different NBI heating pulses which are long (open circles) and short (closed squares) with respect to the alpha thermalization time $(\approx 0.4 \mathrm{~s})$. The data from both agree with model calculations assuming classical alpha-particle confinement and thermalization (solid and dashed lines). The alphas near their birth energy of $3.5 \mathrm{MeV}$ can be seen in the short-pulse case.

Figure 2 shows the results of an experiment designed to test the basic physics of alpha-particle thermalization using the PCX diagnostic [3]. One of these two discharges had a long (1.0 s) DT neutral beam heating (NBI) pulse and corresponding long alpha source duration compared with the alpha thermalization time, while the other had only a very short NBI pulse. The energy spectrum for both cases was measured near the plasma centre immediately after the NBI was turned off. The measured spectrum for the long-duration pulse was consistent with the classical collisional slowing-down model, as calculated by a time-dependent axisymmetric alpha-particle orbit code, FFPT (FokkerPlanck post TRANSP processor) [4]. The spectrum for the short-pulse case was a nonthermalized alpha distribution near the birth energy at $3.5 \mathrm{MeV}$, also as expected from the classical collisional model.

Measurements of the alpha-energy spectrum and radial profile were made near the plasma centre using the Alpha-CHERS diagnostic. The inferred central alpha density agreed with the classical collisional model to within $\pm 30 \%$ [4] and an analysis of the radial profile of the alpha density gave a very low alpha diffusion coefficient of $D<0.1 \mathrm{~m}^{2} \mathrm{~s}^{-1}$ [5], similar to the inference from the alpha loss data of figure 1 . 


\section{Alpha transport due to non-axisymmetric perturbations}

Large-scale non-axisymmetric perturbations occur in tokamaks as a result of the static toroidal field (TF) ripple and the fluctuating plasma-driven MHD activity. Stochastic ripple diffusion (SRD) is predicted when the trapped alpha banana tips fall where the TF ripple strength is above about $\delta_{\mathrm{s}} \approx 0.1 \%$, which roughly corresponds to $r / a>0.5$ in TFTR. Monte Carlo calculations predict the alpha-ripple loss fraction to be $\approx 10 \%$ for standard TFTR plasmas with $R=2.5 \mathrm{~m}, I_{\mathrm{p}}=2.5 \mathrm{MA}$ [6], i.e. larger than the calculated first-orbit loss fraction of $\approx 3 \%$. Collisional effects can also contribute significantly to this alpha loss mechanism $[6,7]$.

The stochastic diffusion threshold due to low- $n$ coherent MHD instabilities is much higher than that for the high- $n$ TF ripple [8] so that MHD modes do not normally cause alpha transport in TFTR. However, transient magnetic reconnection events such as a sawtooth crash or a plasma disruption do cause alpha redistribution and loss.

The radial profile of confined trapped alphas along the outer midplane has been measured using the PCX technique described in section 1. The outer edge of the confined alpha population was located near the predicted SRD boundary and variations in its location were consistent with the SRD model [9]. Trapped alphas undergoing SRD are calculated to be lost just below the outer midplane and alpha loss has been measured there at the expected pitch angle for SRD [10]. Both these results are consistent with the standard SRD model, but the absolute magnitude of the TF ripple loss has not yet been measured on TFTR.

Several types of coherent MHD activity cause alpha loss on TFTR. For example, kinetic ballooning modes $(\mathrm{KBM})$ with $f \approx 100 \mathrm{kHz}$ and $n \approx 6$ at $r / a \approx 0.3$ have caused an alpha loss comparable to the first-orbit alpha loss rate [11]. This KBM-induced alpha loss occurred at a pitch angle near the passing-trapped boundary, as though marginally passing alphas were pushed across the passing-trapped boundary and out to the wall on large trappedparticle orbits. Low-frequency tearing and fishbone modes also cause alpha loss, but the level of such MHD-induced loss is usually less than the normal first-orbit loss (i.e. a few

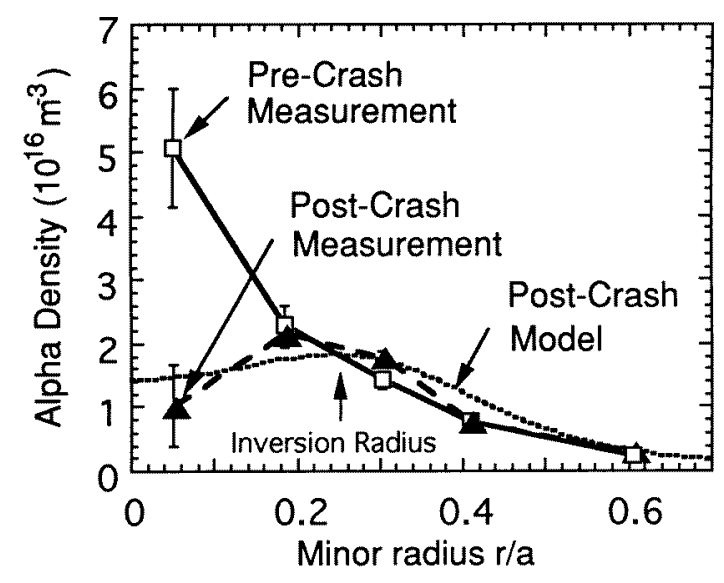

Figure 3. Radial profiles of confined alphas measured in the energy range $0.15-0.6 \mathrm{MeV}$ using the Alpha-CHERS diagnostic. The profile just before the sawtooth crash is very peaked, as expected from the peaked TFTR alpha source. The sawtooth crash significantly redistributes the confined alphas from inside to outside the $q=1$ sawtooth inversion radius. The measured redistribution is consistent with a sawtooth mixing model. 
$\%$ globally), except during the large MHD activity during plasma disruptions [1].

Measurements of the confined alpha distribution before and after a sawtooth crash were made using both confined alpha diagnostics. Results for the Alpha-CHERS measurement are shown in figure 3. The alpha density on axis was reduced by a factor of five after the crash [12], while it increased outside the $q=1$ surface, consistent with a magnetic reconnection model [12] in which the total alpha population was the same before and after the crash. The PCX diagnostic saw a larger increase in the trapped alpha density outside the $q=1$ surface after a sawtooth [13], consistent with a simulation which takes into account the energy change of trapped alphas due to the perturbed electric field during the sawtooth crash [4]. The alpha loss detectors see only a small $\approx 100 \mu$ s burst of alpha loss at the passing-trapped boundary during a sawtooth crash.

\section{Alpha-particle heating and ash build-up}

The global alpha heating effect in TFTR is small because the ratio of alpha heating power to NBI power is typically $\approx 1 \mathrm{MW} / 30 \mathrm{MW}$. However, the local alpha heating fraction near the plasma centre is significantly larger and was measured by carefully comparing electron temperature profiles for a matched series of reproducible DT, TT, and DD discharges [14]. A systematic $\approx 0.6 \mathrm{keV}(\approx 6 \%)$ increase in the central electron temperature was observed in DT compared with similar DD and TT discharges. The expected alpha heating was modelled assuming classical alpha-particle confinement and thermalization, and the results were consistent with the time evolution of the magnitude and radial profile of this measured electron heating, after correcting for the improved plasma confinement with tritium.

The thermal He ash source from the alpha thermalization in TFTR is typically only $\approx 1 \%$ of the plasma ion density per second. This small ash content was measured using absolutely calibrated spectroscopy in a reproducible set of matched DT and DD discharges [15]. The measured profile of thermal He ash was found to be nearly flat at the end of $\mathrm{a} \approx 1.5 \mathrm{~s}$ long, $1 \mathrm{MW}$ alpha heating pulse, with a total He content approximately a factor of two lower than the integrated alpha source. This measurement was successfully modelled using classical fast alpha confinement and thermalization, supplemented by the usual anomalous diffusion and wall recycling coefficients for thermal He. The He ash profile was flat since the thermal He confinement time was shorter than the pulse length, so most of the He ash content was recycled from the wall, i.e. the ash did not accumulate in the plasma core.

\section{Interaction of alpha particles with $R F$ waves}

Experiments have been started on TFTR to control the confinement and thermalization of alpha particles externally using applied RF waves. The simplest RF-alpha interaction occurs when an alpha particle resonates with an ICRF fast wave. This process increases the perpendicular energy of the resonant alphas and can push marginally counter-passing alphas across the passing-trapped boundary. This effect is illustrated in figure 4, which shows the ICRF-induced alpha-particle loss to the bottom of TFTR as a function of the toroidal field $B_{\mathrm{T}}$, which determines the location of the ICRH resonant layer [16]. The alpha loss occurred over a range of $B_{\mathrm{T}}$ consistent with the expected Doppler shifted resonance condition $\omega_{\mathrm{RF}}=\Omega_{\mathrm{c} \alpha}+k_{\|} v_{\alpha}$. Some heating of the lost alpha particles was seen within this resonance region.

One goal of these experiments is to learn how to couple alpha heating directly into the 


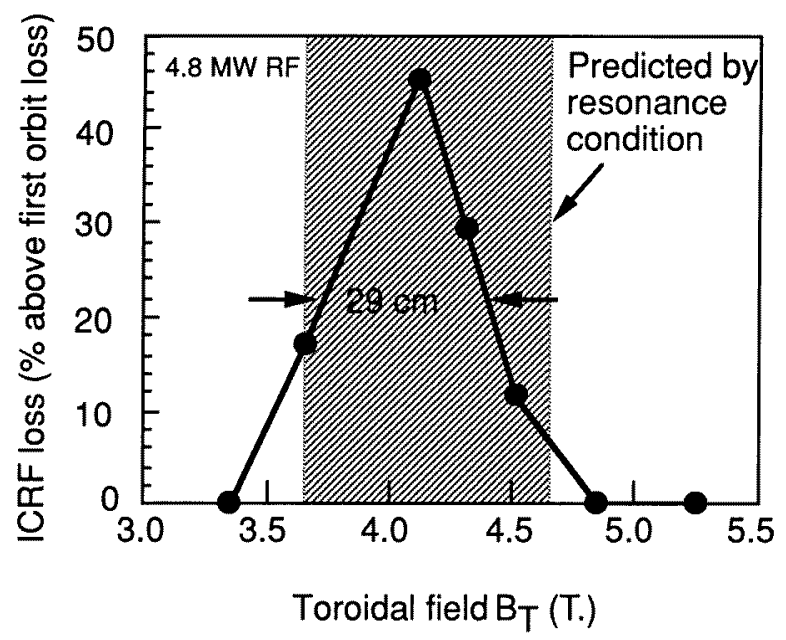

Figure 4. ICRH-induced alpha-particle loss to the bottom scintillator detector as a function of the toroidal field $B_{\mathrm{T}}$. The alpha loss peaks when the Doppler shifted alpha-particle cyclotronresonance layer intersects marginally counter-passing alphas, increasing their perpendicular energy and causing them to be lost across the passing-trapped boundary to the vessel bottom. This alpha loss also peaks at the pitch angle corresponding to the passing-trapped boundary, as expected from this model.

DT fuel ions, i.e. to perform 'alpha channelling' [17]. The first step in this process is to demonstrate RF-induced alpha-particle cooling, which has been predicted for a specific phasing between alphas and high- $k_{\theta}$ ion Bernstein waves (IBWs) [18]. Although the basic wave-particle interaction heats and cools randomly, a net cooling can occur when the cooled alphas move outwards to a sink at the wall. Preliminary experiments have been performed on TFTR using 1-2 MW of IBWs created by mode conversion of ICRH fast waves. A clear IBW-induced increase in the beam ion loss and beam ion heating to $\approx 2 \mathrm{MeV}$ have been observed [15], but the required conditions for alpha cooling have not yet been obtained.

\section{Alpha-particle instabilities}

A search for the alpha-driven toroidal Alfvén eigenmodes (TAE) was made in high fusion power DT discharges [19], and also in special DT TAE experiments at lower power [20-22]. However, until recently, there was no clear sign of an alpha-driven TAE mode or beta-induced Alfvén eigenmode (BAE) [23] was seen in NBI heated plasmas with up to $\leqslant 2 \mathrm{MW}$ of alpha heating power, corresponding to $\beta_{\alpha}(0) \leqslant 0.3 \%$.

Theoretical analysis of the TAE stability for these cases suggested that the instability threshold could be lowered by raising $q(0)$ [24], or by reducing beam ion Landau damping [25]. Subsequent analysis predicted that the threshold for core-localized TAEs could be further lowered by weakening the magnetic shear at high $q(0)$ [26]. This motivated an experiment to look for TAEs after the turn-off of NBI in a DT discharge with high $q(0)$ and weak magnetic shear.

Alpha-driven TAEs were seen clearly for the first time in that experiment, as illustrated in figure 5 [27]. The external magnetic loops detected a magnetic fluctuation which matched the expected TAE frequency in DT plasmas, with no similar mode in DD plasmas at equal beam power and similar plasma parameters. This new mode was observed only after the 


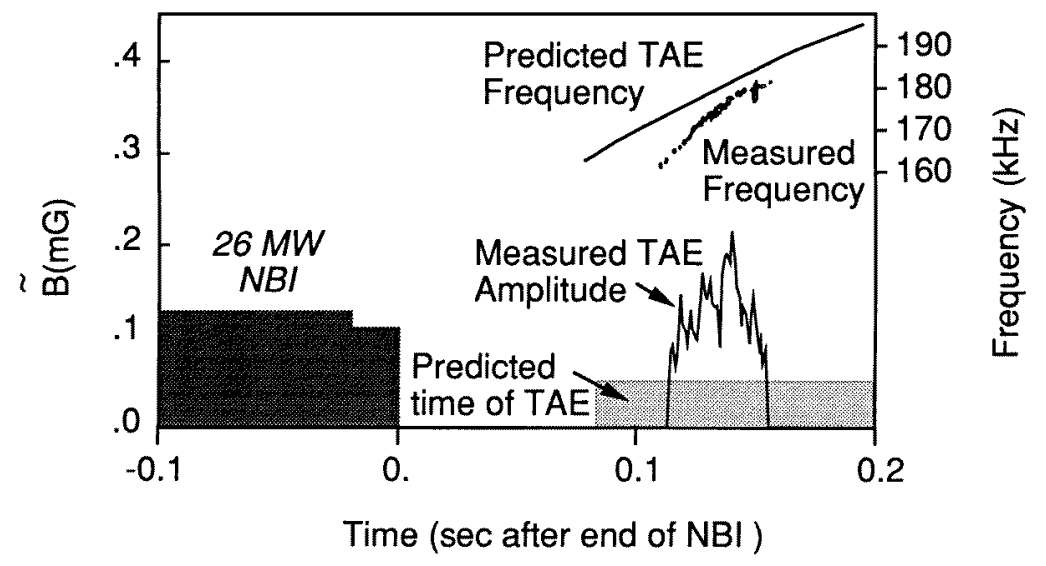

Figure 5. Alpha-driven TAE mode in TFTR occurring $\approx 0.1 \mathrm{~s}$ after neutral beam injection in a DT discharge with weak central magnetic shear. The frequency is consistent with the densitydependent TAE frequency, and mode timing is roughly consistent with the theoretical prediction based on the beam ion Landau damping model. The amplitude of the mode is very small, so it caused no observable alpha-particle loss. No such mode occurred in otherwise similar DD discharges.

beam ions had thermalized and before the alphas had thermalized, as predicted by the theory of beam ion damping. The radial location has been determined by reflectometry to be near the maximum alpha pressure gradient at $r / a \approx 0.3$, as expected.

The amplitude of this alpha-driven TAE mode is smaller than the previously observed NBI-driven TAE modes in TFTR (at very low field). However, the alpha-driven TAE occurs at a much lower fast ion pressure of $\beta_{\alpha}(0) \approx 0.1 \%$, corresponding to $\leqslant 1 \mathrm{MW}$ peak alpha power. The alpha pressure threshold for this TAE mode is at least a factor of three below that obtained in the highest-powered DT discharges which had no signs of TAE modes after NBI. Clearly, the threshold is sensitive to factors other than just the alpha pressure, such as the beam ion damping and the $q(r)$ profile. No alpha loss due to this small TAE has yet been observed.

The only other alpha-driven instability seen in TFTR is a very weak emission in the ioncyclotron emission (ICE) range of frequencies, which has been attributed to alpha-particle instabilities driven by the anisotropic alpha-particle distribution at the plasma edge [28, 29]. Qualitatively similar ICE is also observed in DD discharges at much lower fusion product level, apparently driven by the small $3 \mathrm{MeV}$ proton density. There has been no alpha loss or any other plasma effect on the plasma due to this instability.

\section{Discussion}

These experiments on TFTR have provided a first look at the behaviour of DT alpha particles in a tokamak plasma. The confinement and thermalization of alphas appears to be classical near the centre of MHD-quiescent TFTR discharges, and the influence of non-axisymmetric perturbations such as MHD and TF ripple loss seem to be relatively small.

It is interesting that high-energy alpha particles in TFTR can have a diffusion coefficient up to 10 times smaller than the thermal ions. This has been explained by the 'orbit averaging' of small-scale plasma turbulence by the large orbits of the fast alphas [30, 31], 
since $k_{\perp} \rho_{\alpha} \approx 3$, where $k_{\perp}$ is the perpendicular wavenumber of the turbulence and $\rho_{\alpha} \approx 5 \mathrm{~cm}$ is the alpha gyroradius at $3.5 \mathrm{MeV}$. However, this same transport reduction process may not occur in larger tokamaks if the turbulence size scale increases with the machine size.

The alpha heating and He alpha ash content in TFTR are consistent with classical fast alpha confinement and thermalization, but these measurements are difficult and uncertain by about $\pm 50 \%$ and $\pm 30 \%$, respectively. Since only a relatively small part of the alpha distribution function has been directly measured, the alpha confinement has not yet been fully accounted for. For example, the effect of coherent MHD activity on confined alphas has not yet been measured, the 2D pattern of alpha loss to the wall is not well known, and the origin of a newly discovered low-energy alpha loss at the bottom of TFTR [32] is not understood.

A clear alpha interaction with ICRF fast waves has been observed, and the physics of this interaction has been identified. Direct external control over alpha thermalization and transport is possible using such RF interactions, but has not yet been realized in TFTR.

Most of the MHD- and RF-induced alpha-particle loss measured in TFTR occurs at the passing-trapped boundary, at which point alphas near the core of TFTR can be lost to the wall in a single large step due to a relatively small perturbation in the passing particle orbit. This first-orbit loss region will be smaller in a reactor-sized tokamak, but the analogous ripple-loss region could be similarly sensitive to wave-induced internal diffusion of alphas.

So far alpha-particle-driven instabilities have not caused a serious problem with alphaparticle confinement in TFTR, although a small alpha-driven TAE mode has been observed at a very low $\beta_{\alpha} \approx 0.1 \%$. It is not yet clear what the eventual effect of an alpha-driven TAE will be on a burning tokamak plasma with a much higher alpha pressure, so experiments will continue to test the physics of the instability drive, damping, and the consequent alpha transport effects.

\section{Acknowledgments}

We thank H Furth, J Hosea, D Meade and K M Young for their support for this work, which was performed under US DOE Contract No DE-AC02-76-CH03073.

\section{References}

[1] Zweben S J et al 1995 Nucl. Fusion 35893

[2] Heidbrink W W and Sadler G 1994 Nucl. Fusion 34535

[3] Medley S S 1996 Plasma Phys. Control. Fusion 381

[4] Gorelenkov N et al 1996 Princeton Plasma Physics Laboratory Report PPPL-3184 Nucl. Fusion submitted

[5] McKee G et al 1995 Phys. Rev. Lett. 75649

[6] Redi M H et al 1995 Nucl. Fusion 351191

[7] Goloborod'ko V et al 1996 Proc. 23rd EPS Conf. Controlled Fusion and Plasma Physics (Kiev) p A74

[8] White R B and Mynick H E 1989 Fluids B 1980

[9] Duong $\mathrm{H}$ et al 1997 Nucl. Fusion to be published

[10] Zweben S J et al 1995 Nucl. Fusion 351445

[11] Chang Z et al 1996 Phys. Rev. Lett. 761071

[12] Stratton B C et al 1996 Nucl. Fusion 361586

[13] Petrov M et al 1996 16th Int. Conf. Nuclear Fusion Research (Montreal)

[14] Taylor G et al 1996 Phys. Rev. Lett. 762722

[15] Synakowski E et al 1995 Phys. Rev. Lett. 753689

[16] Darrow D S et al 1997 Nucl. Fusion to be published

[17] Fisch N et al 1996 16th Int. Conf. Nuclear Fusion Research (Montreal)

[18] Valeo E J and Fisch N J 1994 Phys. Rev. Lett. 733536

[19] Strachan J D et al 1994 Phys. Rev. Lett. 723526 
[20] Batha S H et al 1995 Nucl. Fusion 351463

[21] Wong K L et al 1996 Phys. Rev. Lett. 762286

[22] Zweben S J et al 1996 Nucl. Fusion 36978

[23] Heidbrink W W et al 1996 Nucl. Fusion 361725

[24] Spong D A et al 1995 Nucl. Fusion 351687

[25] Fu G Y et al 1995 Phys. Rev. Lett. 752336

[26] Fu G Y et al 1996 Phys. Plasmas to be published

[27] Nazikian R et al 1996 16th Int. Conf. Nuclear Fusion Research (Montreal)

[28] Dendy R O et al 1995 Nucl. Fusion 351733

[29] Arunasalam W 1997 J. Plasma Phys. to be published

[30] Myra J R et al 1993 Phys. Fluids B 51160

[31] Manfredi G and Dendy R O 1996 Phys. Rev. Lett. 764360

[32] Herrmann H W et al 1997 Nucl. Fusion to be published 